\title{
Genotoxicity in Patients on Long-term Proton Pump Inhibitor Therapy in Korea: A Nested Case-control, Prospective, Pilot Study
}

\author{
Youn I Choi ', Jun-Won Chung ', Dong Kyun Park', Kyoung Oh Kim ', Kwang An Kwon', Yoon Jae Kim', Su Young Kim², Sung-Min Ahn', \\ Mun-Deok Han ${ }^{4}$, Kwang-Pil Ko ${ }^{5}$ \\ Department of Gastroenterology, Gil Medical Center, Gachon University College of Medicine ${ }^{1}$, Incheon, Department of Gastroenterology, \\ Yonsei University Wonju College of Medicine $e^{2}$, Wonju, Department of Hematology-Oncology, Gil Medical Center, Gachon University College of \\ Medicine ${ }^{3}$, Incheon, Division of Research and Development, Bio Research Complex Co., Ltd. ${ }^{4}$, Incheon, Department of Preventive Medicine, \\ Gachon University College of Medicine ${ }^{5}$, Incheon, Korea
}

Background/Aims: Although proton pump inhibitors (PPIs) remain a mainstay for the suppression of gastric acid secretion, long-term PPI use is associated with side effects. However, the genotoxicity associated with long-term PPI use is unclear.

Materials and Methods: This prospective observational pilot study enrolled patients who had been on PPIs for $>1$ year and healthy controls from July 2015 to August 2016. The subjects completed self-report questionnaires pertaining to their drug and medical history, and only those with no medical history and a $\geq 2$-year wash-out period (for drugs other than PPIs) were included. We collected peripheral-blood lymphocytes from long-term PPI users and healthy controls and analyzed the genotoxicity by using the cytokinesis-block micronucleus cytome assay; we also determined the fasting serum levels of pyridoxine, folate, cobalamin, and homocysteine.

Results: Ten long-term PPI users and 40 healthy control subjects were enrolled. The median serum pyridoxine, folate, cobalamin, and homocysteine levels were not significantly different between the groups. The median frequencies of micronuclei (MNi), nucleoplasmic bridges (NPBs), and nuclear buds (Nbuds) per 1,000 binucleated cells, in long-term PPI users and healthy controls, were 30.3 and $16.3(P<0.005), 2.5$ and $1.8(P<0.005)$, and 9.3 and $5.0(P<0.005)$, respectively. Even after adjustment for confounding factors, the OR of the MNi, NPBs, and Nbuds for long-term PPI users compared with healthy control subjects were $14.1(P<0.001)$ $2.0(P=0.001)$, and $1.3(P=0.3)$, respectively.

Conclusions: Long-term PPI use was significantly associated with an increased risk of genotoxicity after adjustment for age, sex, body mass index, medical history, drug history, and the serum levels of vitamins. (Korean J Helicobacter Up Gastrointest Res 2020;20:47-53)

Key Words: Genotoxicity test; Prospective study; Proton pump inhibitors

\section{INTRODUCTION}

Proton pump inhibitors (PPIs) suppress gastric acid secretion and are used to treat gastroesophageal reflux disease and peptic ulcers. ${ }^{1,2}$ Because PPIs are considered effective and are well-tolerated, they are also prescribed for inappropriate conditions. ${ }^{1,2}$

Long-term use of PPIs can cause side effects of varying severity. Unlike the rare and mild side effects of short-term use of PPIs, the potential long-term complications of PPIs

Received: August 6, 2019 Revised: October 29, 2019 Accepted: November 30, 2019 Corresponding author: Jun-Won Chung

Department of Gastroenterology, Gil Medical Center, Gachon University College of Medicine, 21 Namdong-daero 774beon-gil, Namdong-gu, Incheon 21565, Korea Tel: +82-32-460-2193, Fax: +82-32-460-3778, E-mail: junwonchung@hanmail.net This research is funded by the Korean College of Helicobacter and Upper Gastrointestina Research Jeil Fund 2012. However, we declare all author in this study have no potential competing interests. include an increased risk of infection, dementia, chronic kidney disorders, cardiovascular diseases, and cancer., ${ }^{1,3-8}$ However, the mechanisms underlying the long-term side effects of PPIs are unclear, ${ }^{5,6}$ as is their genotoxicity.

Micronuclei (MNi), nucleoplasmic bridges (NPBs), and nuclear buds (Nbuds) in peripheral blood lymphocytes (PBL) are used as biomarkers of chromosomal damage. $\mathrm{MNi}$ is a biomarker of chromosome breakage and/or loss. ${ }^{9-11}$ NPBs indicate DNA strand-break misrepair and/or telomere end fusion. ${ }^{9-11}$ Nbuds are biomarkers of gene amplification and/or elimination of DNA repair complexes. ${ }^{9-11}$ However, the genotoxicity of long-term PPI use is unknown. In this study, we assessed the genotoxicity of long-term PPI use by cytokinesis-block micronucleus cytome (CBMN-Cyt) assay, with adjustment for demographic variables, comorbidities, drug history, and serum vitamin levels.

Copyright $\odot 2020$ Korean College of Helicobacter and Upper Gastrointestinal Research

(a) The Korean Journal of Helicobacter and Upper Gastrointestinal Research is an Open-Access Journal. All articles are distributed under the terms of the Creative Commons Attribution Non-Commercial License (http:// creativecommons.org/licenses/by-nc/4.0) which permits unrestricted non-commercial use, distribution, and reproduction in any medium, provided the original work is properly cited. 


\section{MATERIALS AND METHODS}

The Institutional Review Board of Gil Medical Center approved the study protocol, and the study was registered with the Clinical Research Information Service (KCT0001688).

\section{Definition of long-term PPI users and healthy controls}

This was a nested case-control study performed in the Gil Medical Center from July 2015 to August 2016. In this study, all of the subjects completed self-report questionnaires pertaining to their drug and medical history, and only those with no medical history (other than reflux esophagitis for PPI users) and a $\geq 2$-year drug (other than PPIs) wash-out period were included.

The inclusion criteria for the long-term PPI users ( $>1$ year) were: age 20 80 years, non-smokers (>10 years since quitting smoking), and no drug history within 2 years. The exclusion criteria for the long-term PPI users were as follows: use of supplemental vitamins in the previous 6 months, conditions other than gastroesophageal reflux disease (daily alcohol intake $>35 \mathrm{~g}$ for men or $>28 \mathrm{~g}$ for women, strict vegetarianism, history of cancer, gastrectomy, ileal disease, or pregnancy), or patients who had taken drugs other than PPIs within 2-year period. The patients underwent gastrointestinal endoscopy to detect gastrointestinal disorders other than gastroesophageal reflux.

The healthy controls, who had visited Gil Medical Center for a health check-up, were non-smokers or had quit smoking $>10$ years prior, and had no known disease or drug history within 6 months. The other exclusion criteria were the same as for the long-term PPI users.

Written informed consent was obtained from all subjects prior to participation. The subjects were informed of the study aims and methods, as well as possible side effects.

\section{Laboratory parameters}

Blood samples were obtained from all patients following a 12 hours fast and were collected in serum-separating tubes or $5 \mathrm{mmol} / \mathrm{L}$ ethylenediaminetetraacetic acid tubes. Blood samples were centrifuged for 15 minutes at 3,300 rpm and stored at $-20^{\circ} \mathrm{C}$ until analysis. Pyridoxine analysis was performed by high-performance liquid chromatography. Folate and cobalamin levels were measured by radioimmunoassay, and that of homocysteine by chemiluminescence immunoassay.

\section{CBMN assay}

We analyzed genotoxicity by CBMN-Cyt assay in PBL from long-term PPI users and healthy controls, and determined the fasting serum levels of pyridoxine, folate, cobalamin, and homocysteine. PBLs were isolated from the blood samples and subjected to CBMN-Cyt assays for $\mathrm{MNi}$ (biomarker of chromosome breakage or loss), NPBs (biomarker of DNA mis-repair), and Nbuds (biomarker of elimination of DNA from the nucleus or DNA repair complexes). ${ }^{12-15}$ Blood samples were deidentified and the examiners were blinded to the experimental procedures.

PBLs were cultured aseptically in class II biological safety cabinets $^{12,15-17}$ and phytohemagglutinin (Remel R 30,852,701; Thermo Fisher, Waltham, MA, USA) was added to stimulate mitotic division. ${ }^{15}$ Forty-four hours later, cytochalasin-B (C6762; Sigma-Aldrich, St. Louis, MO, USA) was added to the cultures to capture cells at the binucleated stage (i.e., after completion of one nuclear division). ${ }^{15}$ Next, cytochalasin-B was added and 28 hours later the cells were harvested using a cyto-centrifuge (Thermo cytospin4; Thermo Fisher), fixed in methanol, and stained with Hemacolor ${ }^{\mathbb{B}}$ (111,661; Merck, Kenilworth, NJ, USA).

After staining, the frequencies of MNi, NPBs, and Nbuds per 1,000 binucleated cells (BNCs) were evaluated under a light microscope ( $\times 100$ magnification) according to established scoring criteria. ${ }^{12,16,17}$ Two examiners scored each sample twice and were blinded according to Fenech's criteria. The coefficient of variation between the duplicates was $11.8 \pm 8.2$, and that between the two scorers was $8.8 \pm 3.4$. The average frequencies of MNi, NPBs, and Nbuds per 1,000 BNCs were calculated. ${ }^{12,16,17}$ The nuclear division index was determined from the scores of 500 BNCs using the formula: nuclear division index $=(\mathrm{M} 1+2 \mathrm{M} 2+3 \mathrm{M} 3+4 \mathrm{M} 4) / \mathrm{N}$, where M1 M4 is the number of cells with 1 4 nuclei and $\mathrm{N}$ is the total number of viable cells scored (excluding necrotic 
and apoptotic cells). ${ }^{12,16,17}$

\section{Statistical analysis}

We used SPSS software version 20.0 (IBM Corp., Armonk, NY, USA) for data analysis. Categorical variables were expressed as frequencies and percentages and were compared by Fisher's exact test. Non-parametric data were presented as medians with interquartile ranges and were analyzed by the Mann-Whitney or Kruskal-Wallis test. To identify significant differences between the groups, non-parametric

Table 1. Clinical Characteristics of the Subjects

\begin{tabular}{lcc}
\hline & $\begin{array}{c}\text { PPI long-term users } \\
(\mathrm{n}=10)\end{array}$ & $\begin{array}{c}\text { Controls } \\
(\mathrm{n}=40)\end{array}$ \\
\hline Male/female & $3 / 7$ & $25 / 15$ \\
Age (years) & 58.0 & 36.3 \\
BMI & $23.6 \pm 3.2$ & $22.8 \pm 3.8$ \\
$\begin{array}{l}\text { Duration of PPI use } \\
\text { (months) }\end{array}$ & \\
$12 \sim 18$ & $6(60.0)$ & \\
$19 \sim 24$ & $2(20.0)$ & \\
$>25$ & $2(20.0)$ & \\
Reasons for PPI use & & \\
Reflux disease & $10(100.0)$ &
\end{tabular}

Values are presented as mean \pm standard deviation or $\mathrm{n}(\%)$.

PPI, proton pump inhibitor; BMI, body mass index. categorical variables were analyzed by Mann-Whitney test. A linear regression model was used to analyze the relationship between genomic damage (CBMN-Cyt assay scores) and the independent variables (age, sex, BMI, and levels of pyridoxine, folate, cobalamin, and homocysteine. A value of $P<0.05$ was considered indicative of significance.

\section{RESULTS}

\section{Baseline characteristics}

Ten consecutive long-term PPI users and 40 healthy controls were analyzed; their clinical characteristics were shown in Table 1. All of the long-term PPI users were being treated for reflux esophageal disorders. Four (40\%) of the long-term PPI users had been on PPIs for $>19$ months.

\section{Serum levels of pyridoxine, folate, cobalamin, and homocysteine}

There were no significant differences between the long-term PPI users and healthy controls in the serum levels of pyridoxine, folate, cobalamin, and homocysteine (Table 2).

Table 2. Pyridoxine, Folate, Cobalamin, and Homocysteine Levels in the Subjects

\begin{tabular}{lccc}
\hline & PPI long-term users $(\mathrm{n}=10)$ & Controls $(\mathrm{n}=40)$ & $P$-value \\
\hline Pyridoxine $(\mathrm{nmol} / \mathrm{L})$ & $58.5(42.2 \sim 51.9)$ & $61.7(41.7 \sim 82.9)$ & 0.9 \\
Folate $(\mathrm{ng} / \mathrm{mL})$ & $7.5(5.3 \sim 12.2)$ & $6(4.6 \sim 8.4)$ & 0.2 \\
Cobalamin $(\mathrm{pg} / \mathrm{mL})$ & $645.3(438.3 \sim 792.5)$ & $529.1(408.6 \sim 699.6)$ & 0.4 \\
Homocysteine $(\mathrm{umol} / \mathrm{L})$ & $8.9(8.2 \sim 10.5)$ & $10.5(8.4 \sim 12.4)$ & 0.2 \\
\hline
\end{tabular}

Values are presented as median (interquartile range).

PPI, proton pump inhibitor.

Table 3. Cytokinesis-block Micronucleus Cytome Assay Score according to Proton Pump Inhibitor Use

\begin{tabular}{lccc}
\hline & PPI long-term users $(\mathrm{n}=10)$ & Control $(\mathrm{n}=40)$ & $P$-value \\
\hline MNi & $30.3(21.2 \sim 31.9)$ & $16.3(12.3 \sim 32.5)$ & 0.003 \\
NPBs & $2.5(2.2 \sim 2.6)$ & $1.8(1.0 \sim 2.7)$ & 0.01 \\
Nbuds & $9.3(9.2 \sim 9.3)$ & $5.0(4.5 \sim 8.2)$ & 0.2 \\
\hline
\end{tabular}

Values are presented as median (range).

PPI, proton pump inhibitor; MNi, micronuclei; NPBs, nucleoplasmic bridges; Nbuds, nuclear buds. 


\section{Frequencies of BNCs with $\mathrm{MNi}$, NPBs, and Nbuds}

The frequencies of BNCs with $\mathrm{MNi}$ were significantly higher in the long-term PPI users than in the controls (30.3 [21.2 31.9] vs. 16.3 [12.3 32.5]; $P=0.003$ ), as were the frequencies of BNCs with NPBs (2.5 [2.2 2.6] vs. 1.8 [1.0 2.7]; $P=0.01$ ). The long-term PPI users showed higher frequencies of BNCs with Nbuds than the controls but statistically insignificant (9.3 [9.2 9.3] vs. 5.0 [4.5 8.2]; $P=0.2$ ) (Table 3).

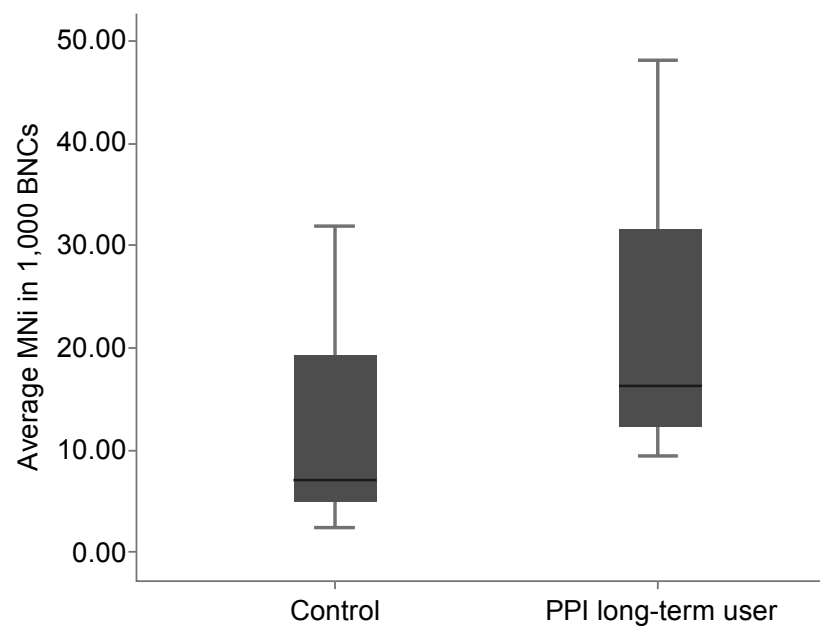

Fig. 1. Average micronuclei (MNi) in 1,000 binucleated cells (BNCs). PPI, proton pump inhibitor.

\section{Univariate and multivariate analyses}

In univariate analysis, long-term PPI use was statistically significantly associated with the increased frequencies of $\mathrm{MNi}$, NPBs and Nbuds. Increased frequencies of MNi were significantly associated with PPI long-term use after adjusting the level of the age, male, BMI, and vitamin levels (OR 14.1, $P<0.001$ ) (Fig. 1 and Table 4). Also in NPBs, the increased frequencies of the NPBs were associated with PPI long-term use even after adjusting the demographic factors including age, gender, BMI, and vitamin level including pyridoxine, folate, cobalamine, and homocysteine (OR 2.0, $P<0.001$ ) (Table 5). In Nbuds, the increased levels of frequencies of Nbuds were associated with PPI long-term use (OR 1.3, $P=0.3)$ (Table 6).

\section{DISCUSSION}

We reported that long-term PPI use was associated with increased frequencies of genetic damages (MNi, NPBs, and Nbuds). The association between long-term PPI use and genotoxicity retained its significance after adjustment for possible confounding factors. To our knowledge, this is the first study to evaluate the genotoxicity of long-term use of PPIs by CBMN-Cyt assay using human PBLs.

The multi-endpoint CBMN-Cyt assay enables assessment of genotoxicity and DNA dpamage. In this study we used three biomarkers (MNi, NPBs, and Nbuds) to assess the genotoxicity of long-term PPI use. Abnormal levels of MNi, NPBs, and Nbuds in PBLs were indicative of genetic instability.

Table 4. Univariate and Multivariate Analyses of Micronuclei and Genomic Damage-associated Factors

\begin{tabular}{|c|c|c|c|c|c|c|}
\hline & \multicolumn{3}{|c|}{ Univariate analysis } & \multicolumn{3}{|c|}{ Multivariate analysis } \\
\hline & Estimate & SE & $P$-value & Estimate & SE & $P$-value \\
\hline Age (years) & 0.4 & 0.07 & $<0.001$ & 8.4 & 3.5 & 0.02 \\
\hline Male & 8.8 & 3.0 & 0.005 & 3.7 & 2.8 & 0.2 \\
\hline BMI & -0.002 & 0.6 & 0.9 & -0.4 & 0.4 & 0.3 \\
\hline PPI long-term use & 10.7 & 3.7 & 0.006 & 14.1 & 3.4 & $<0.001$ \\
\hline Pyridoxine (nmol/L) & 0.06 & 0.03 & 0.03 & 0.007 & 0.02 & 0.8 \\
\hline Folate $(\mathrm{ng} / \mathrm{mL})$ & 1.6 & 0.6 & $<0.001$ & 1.4 & 0.5 & 0.01 \\
\hline Cobalamin (pg/mL) & 0.01 & 0.006 & 0.04 & -0.009 & 0.008 & 0.2 \\
\hline Homocysteine $(\mu \mathrm{mol} / \mathrm{L})$ & -0.4 & 0.6 & 0.5 & 0.7 & 0.5 & 0.2 \\
\hline
\end{tabular}

SE, standard error; BMI, body mass index; PPI, proton pump inhibitor. 
Age, ${ }^{11,18}$ gender, ${ }^{18}$ smoking status, ${ }^{18,19}$ vitamin status, ${ }^{19}$ seasonal variations, ${ }^{15}$ lifestyle factors, ${ }^{15,18,20}$ drugs and transition metals, ${ }^{21}$ and occupational and environmental exposure to genotoxic substances ${ }^{11,22}$ has been associated with an increased risk of genotoxicity. Also, a variety of diseases, ${ }^{11,22-24}$ including Down syndrome, congenital anomalies, aplastic anemia, and solid cancers (e.g., breast cancers) has been associated with genomic instability. ${ }^{22,25}$ Therefore, we evaluated the aforementioned factors to assess the genotoxicity of long-term PPI use, and included only patients with no previous medical or drug history within 2 years.

Increased frequencies of MNi, NPBs, and Nbuds, used as markers of carcinogenic events, are associated with an increased risk of cancer. ${ }^{9}$ Genotoxicity is associated with an increased risk of carcinogenesis. ${ }^{11,23}$ El-Zein et al. ${ }^{11}$ reported that increased genotoxicity was associated with the development of lung cancer (area under the curve for lung cancer,
0.979; 95\% CI, 0.959 0.990). ${ }^{11,23}$ Based on these reports, patients with abnormal CBMN-Cyt assay findings should be carefully monitored during follow-up.

There have been several reports pertaining to the association of long-term PPI use and increased risk of cancer development in clinical studies. Chien et al. ${ }^{7}$ reported that PPIs were associated with an increased risk of periampullary cancer in a dose- and duration-dependent manner (OR, 1.35 [95\% CI, 1.16 1.57]). Chu et al..$^{26}$ reported that PPI use was associated with decreased risk of progression free survival and overall survival in patients with capecitabine treatment in advanced gastroesophageal cancer as a randomized clinical trial (progression free survival, hazard ratio: $1.55, P<0.001$; overall survival, hazard ratio: 1.41 , $P<0.001$ ). Sun et al. ${ }^{24}$ showed that concomitant administration of PPIs and capecitabine was associated with an increased risk of recurrence of early colorectal cancer (OR

Table 5. Univariate and Multivariate Analyses of Nucleoplasmic Bridges and Genomic Damage-associated Factors

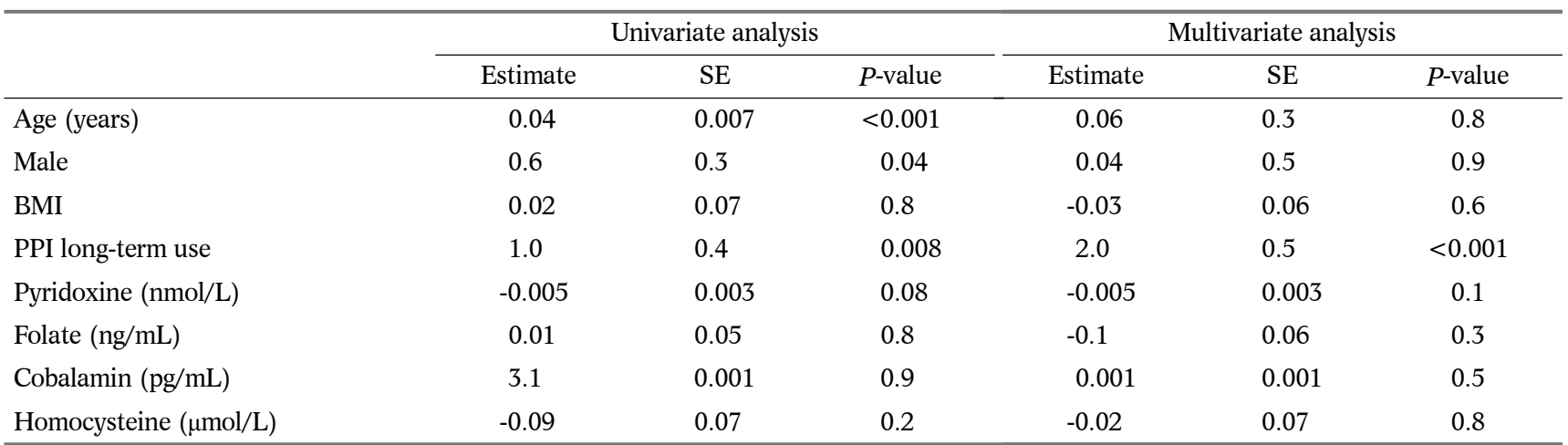

SE, standard error; BMI, body mass index; PPI, proton pump inhibitor.

Table 6. Univariate and Multivariate Analyses of Nuclear Buds and Genomic Damage-associated Factors

\begin{tabular}{|c|c|c|c|c|c|c|}
\hline & \multicolumn{3}{|c|}{ Univariate analysis } & \multicolumn{3}{|c|}{ Multivariate analysis } \\
\hline & Estimate & SE & $P$-value & Estimate & SE & $P$-value \\
\hline Age (years) & 0.08 & 0.02 & $<0.001$ & -0.4 & 1.1 & 0.7 \\
\hline Male & 1.6 & 0.8 & 0.04 & -0.4 & 1.1 & 0.8 \\
\hline BMI & 0.02 & 0.1 & 0.9 & -0.005 & 0.1 & 0.9 \\
\hline PPI long-term use & 0.8 & 1.0 & 0.4 & 1.3 & 1.1 & 0.3 \\
\hline Pyridoxine (nmol/L) & 0.006 & 0.006 & 0.4 & -0.002 & 0.008 & 0.8 \\
\hline Folate (ng/mL) & 0.3 & 0.09 & 0.005 & 0.2 & 0.2 & 0.2 \\
\hline Cobalamin (pg/mL) & 0.002 & 0.002 & 0.2 & -0.001 & 0.003 & 0.8 \\
\hline Homocysteine $(\mu \mathrm{mol} / \mathrm{L})$ & -0.3 & 0.1 & 0.03 & -0.2 & 0.2 & 0.3 \\
\hline
\end{tabular}

SE, standard error; BMI, body mass index; PPI, proton pump inhibitor. 
1.89, $P=0.03)$. However, the mechanisms underlying the association between PPI use and carcinogenesis or poor cancer-related outcomes are unclear. Although our study did not determine the association of long-term PPI use with clinical outcomes, genetic instability might be associated with the side effects associated with such in long-term use.

This study had several limitations. First, it involved only one tertiary center and included a small population, which may have resulted in referral bias. Because we included only patients with no medical or drug history for 2 years, the inclusion criteria were robust; however, this precluded enrollment of a larger number of subjects. Nevertheless, the long-term users of PPIs exhibited greater genotoxicity (larger numbers of MNi, NPBs, and Nbuds) than the healthy controls. Second, another concern in this study is the selection bias. While PPI users were enrolled among the patients who visited the gastroenterology outpatient clinic of a tertiary hospital, control groups were enrolled among those who visited the hospital for a health check-up. Even if we had every effort to avoid the bias adjusting for confounders, biases might be issue in this study. Third, we showed not the causal relationship but the associations between the genotoxicity and the long-term PPI use. Further larger and long-term prospective studies are needed. Fourth, we analyzed only Asian patients, so the conclusions cannot be generalized to other ethnic groups. Fifth, in this study, we did not show the implications of the genotoxicity induced by the long-term PPI use. Even though genotoxicity has been known to be related with diversity of diseases, the direct relations with the genotoxicity related with long-term PPI use should be further studied.

Despite of the aforementioned limitations, this is the first study to reveal the association between genotoxicity and the long-term PPI use. Since, PPIs are generally regarded as the 'safe medications' among patients and some physicians, they are sometimes improperly long-term used. This study might make physicians to care patients on PPIs with cautions, and further large and prospective long-term follow-up study is needed to robust our results.

In conclusion, we delineated that long-term PPI use might be associated with increased risk of genotoxicity. Even though further larger-scale study is needed to robust our results, physicians should be careful in inappropriate use of PPI.

\section{CONFLICT OF INTEREST}

No potential conflict of interest relevant to this article was reported.

\section{ORCID}

$\begin{array}{ll}\text { Youn I Choi } & \text { (D https://orcid.org/0000-0001-6561-6752 } \\ \text { Jun-Won Chung } & \text { (D https://orcid.org/0000-0002-0869-7661 } \\ \text { Dong Kyun Park } & \text { (D https://orcid.org/0000-0002-2862-6641 } \\ \text { Kyoung Oh Kim } & \text { (D https://orcid.org/0000-0002-5365-2550 } \\ \text { Kwang An Kwon } & \text { (D) https://orcid.org/0000-0002-2947-2111 } \\ \text { Yoon Jae Kim } & \text { (D) https://orcid.org/0000-0001-8477-6823 } \\ \text { Su Young Kim } & \text { (D) https://orcid.org/0000-0002-6486-8445 } \\ \text { Sung-Min Ahn } & \text { (D) https://orcid.org/0000-0002-5031-1929 } \\ \text { Mun-Deok Han } & \text { (D) https://orcid.org/0000-0002-1035-2355 } \\ \text { Kwang-Pil Ko } & \text { (D) https://orcid.org/0000-0002-7788-2887 }\end{array}$

\section{REFERENCES}

1. Eusebi LH, Rabitti S, Artesiani ML, et al. Proton pump inhibitors: Risks of long-term use. J Gastroenterol Hepatol 2017;32: 1295-1302.

2. Thomson AB, Sauve MD, Kassam N, Kamitakahara H. Safety of the long-term use of proton pump inhibitors. World J Gastroenterol 2010;16:2323-2330.

3. Tran-Duy A, Spaetgens B, Hoes AW, de Wit NJ, Stehouwer CD. Use of proton pump inhibitors and risks of fundic gland polyps and gastric cancer: systematic review and meta-analysis. Clin Gastroenterol Hepatol 2016;14:1706-1719.e5.

4. Freedberg DE, Haynes K, Denburg MR, et al. Use of proton pump inhibitors is associated with fractures in young adults: a population-based study. Osteoporos Int 2015;26:2501-2507.

5. Thanan R, Ma N, Iijima K, et al. Proton pump inhibitors suppress iNOS-dependent DNA damage in Barrett's esophagus by increasing Mn-SOD expression. Biochem Biophys Res Commun 2012; $421: 280-285$.

6. Qorraj-Bytyqi H, Hoxha R, Sadiku S, et al. Proton pump inhibitors intake and iron and vitamin b12 status: a prospective comparative study with a follow up of 12 months. Open Access Maced J Med Sci 2018;6:442-446.

7. Chien LN, Huang YJ, Shao YH, et al. Proton pump inhibitors and risk of periampullary cancers--a nested case-control study. Int J Cancer 2016;138:1401-1409.

8. Song T, Jeon HK, Hong JE, et al. Proton pump inhibition enhan- 
ces the cytotoxicity of paclitaxel in cervical cancer. Cancer Res Treat 2017;49:595-606.

9. Benassi B, Leleu R, Bird T, Clifton P, Fenech M. Cytokinesisblock micronucleus cytome assays for the determination of genotoxicity and cytotoxicity of cecal water in rats and fecal water in humans. Cancer Epidemiol Biomarkers Prev 2007;16: 2676-2680.

10. Rodrigues MA, Beaton-Green LA, Wilkins RC, Fenech MF. The potential for complete automated scoring of the cytokinesis block micronucleus cytome assay using imaging flow cytometry. Mutat Res Genet Toxicol Environ Mutagen 2018;836(Pt A):53-64.

11. El-Zein RA, Fenech M, Lopez MS, Spitz MR, Etzel CJ. Cytokinesis-blocked micronucleus cytome assay biomarkers identify lung cancer cases amongst smokers. Cancer Epidemiol Biomarkers Prev 2008;17:1111-1119.

12. Thomas P, Fenech M. Cytokinesis-block micronucleus cytome assay in lymphocytes. Methods Mol Biol 2011;682:217-234.

13. Fenech M. Cytokinesis-block micronucleus cytome assay. Nat Protoc 2007;2:1084-1104.

14. Dhillon VS, Yeoh E, Salisbury C, et al. Cytokinesis block micronucleus cytome (CBMN Cyt) assay biomarkers and their association with radiation sensitivity phenotype in prostate cancer cases and DNA repair gene hOGG1 (C1245G) polymorphism. Environ Mol Mutagen 2018;59:813-821.

15. Gajski G, Gerić M, Oreščanin V, Garaj-Vrhovac V. Cytokinesisblock micronucleus cytome assay parameters in peripheral blood lymphocytes of the general population: contribution of age, sex, seasonal variations and lifestyle factors. Ecotoxicol Environ Saf 2018;148:561-570.

16. Pastor S, Rodríguez-Ribera L, Corredor Z, et al. Levels of DNA damage (micronuclei) in patients suffering from chronic kidney disease. Role of GST polymorphisms. Mutat Res Genet Toxicol Environ Mutagen 2018;836(Pt A):41-46.

17. Hong SH, Szili EJ, Fenech M, Gaur N, Short RD. Genotoxicity and cytotoxicity of the plasma jet-treated medium on lymphoblastoid WIL2-NS cell line using the cytokinesis block micronucleus cytome assay. Sci Rep 2017;7:3854.
18. Cho NY, Kim KW, Kim KK. Genomic health status assessed by a cytokinesis-block micronucleus cytome assay in a healthy middle-aged Korean population. Mutat Res 2017;814:7-13.

19. Gabriel HE, Crott JW, Ghandour H, et al. Chronic cigarette smoking is associated with diminished folate status, altered folate form distribution, and increased genetic damage in the buccal mucosa of healthy adults. Am J Clin Nutr 2006;83: 835-841.

20. Kirsch-Volders M, Fenech M, Bolognesi C. Validity of the lymphocyte cytokinesis-block micronucleus assay (L-CBMN) as biomarker for human exposure to chemicals with different modes of action: a synthesis of systematic reviews. Mutat Res Genet Toxicol Environ Mutagen 2018;836(Pt A):47-52.

21. Mesic A, Nefic H. Assessment of the genotoxicity and cytotoxicity in environmentally exposed human populations to heavy metals using the cytokinesis-block micronucleus cytome assay. Environ Toxicol 2015;30:1331-1342.

22. Salimi M, Eskandari E. Association of elevated peripheral blood micronucleus frequency and Bmi-1 mRNA expression with metastasis in Iranian breast cancer patients. Asian Pac J Cancer Prev 2018;19:2723-2730.

23. El-Zein RA, Schabath MB, Etzel CJ, Lopez MS, Franklin JD, Spitz MR. Cytokinesis-blocked micronucleus assay as a novel biomarker for lung cancer risk. Cancer Res 2006;66:6449-6456.

24. Sun J, Ilich AI, Kim CA, et al. Concomitant administration of proton pump inhibitors and capecitabine is associated with increased recurrence risk in early stage colorectal cancer patients. Clin Colorectal Cancer 2016;15:257-263.

25. George A, Venkatesan S, Ashok N, Saraswathy R, Hande MP. Assessment of genomic instability and proliferation index in cultured lymphocytes of patients with Down syndrome, congenital anomalies and aplastic anaemia. Mutat Res Genet Toxicol Environ Mutagen 2018;836(Pt A):98-103.

26. Chu MP, Hecht JR, Slamon D, et al. Association of proton pump inhibitors and capecitabine efficacy in advanced gastroesophageal cancer: secondary analysis of the TRIO-013/LOGiC randomized clinical trial. JAMA Oncol 2017;3:767-773. 